\title{
Using Backscattering to Enhance Efficiency in Neutron Detectors
}

Kittelmann, T.; Kanaki, K.; Klinkby, Esben Bryndt; Cai, Xiao Xiao; Cooper-Jensen, C. P.; Hall-Wilton, R.

Published in:

IEEE Transactions on Nuclear Science

Link to article, DOI:

10.1109/TNS.2017.2695404

Publication date:

2017

Document Version

Publisher's PDF, also known as Version of record

Link back to DTU Orbit

Citation (APA):

Kittelmann, T., Kanaki, K., Klinkby, E. B., Cai, X. X., Cooper-Jensen, C. P., \& Hall-Wilton, R. (2017). Using

Backscattering to Enhance Efficiency in Neutron Detectors. IEEE Transactions on Nuclear Science, 64(6), 15621573. https://doi.org/10.1109/TNS.2017.2695404

\section{General rights}

Copyright and moral rights for the publications made accessible in the public portal are retained by the authors and/or other copyright owners and it is a condition of accessing publications that users recognise and abide by the legal requirements associated with these rights.

- Users may download and print one copy of any publication from the public portal for the purpose of private study or research.

- You may not further distribute the material or use it for any profit-making activity or commercial gain

- You may freely distribute the URL identifying the publication in the public portal 


\title{
Using Backscattering to Enhance Efficiency in Neutron Detectors
}

\author{
T. Kittelmann, K. Kanaki, E. Klinkby, X. X. Cai, C. P. Cooper-Jensen, and R. Hall-Wilton
}

\begin{abstract}
The principle of using strongly scattering materials to recover efficiency in detectors for neutron instruments, via backscattering of unconverted thermal neutrons, is discussed in general. The feasibility of the method is illustrated through Geant 4-based simulations involving thermal neutrons impinging on a specific setup with a layer of polyethylene placed behind a single-layered boron-10 thin-film gaseous detector. The results show that detection efficiencies can be as much as doubled in the most ideal scenario, but with associated adverse contributions to spatial and timing resolutions of, respectively, centimeters and tens of microseconds. Potential mitigation techniques to contain the impact on resolution are investigated and are found to alleviate the issues to some degree, at a cost of reduced gain in efficiency.
\end{abstract}

Index Terms-Backscattering, boron-10, geant4, Monte Carlo simulations, neutron detectors, polyethylene (PE).

\section{INTRODUCTION}

$\mathbf{T}$ HE ongoing construction of the European Spallation Source (ESS) [1], [2] has initiated significant development of novel neutronic technologies in the past 5 years. The performance requirements for neutron instruments at the ESS, in particular the unprecedented cold and thermal neutron brightness and flux expected from the source, are very challenging for detector technologies currently available. The designs for neutron detectors presently in operation at neutron scattering facilities have seen only incremental improvements over the past decade [2]-[6] and are reaching fundamental performance limits; this has made research into alternative neutron detectors very topical.

Detection of neutrons with subelectronvolt kinetic energies must necessarily proceed through destructive nuclear processes in which energetic secondaries are released and detected themselves. Only a few stable isotopes such as ${ }^{3} \mathrm{He},{ }^{10} \mathrm{~B},{ }^{6} \mathrm{Li}$,

Manuscript received September 3, 2015; revised September 30, 2016 and February 11, 2017; accepted April 13, 2017. Date of publication April 18, 2017; date of current version June 26, 2017. This work was supported by the EU Horizon 2020 framework through the BrightnESS Project under Project 676548 and the SoNDe Project under Project 654124. (Corresponding author: Thomas Kittelmann.)

T. Kittelmann and K. Kanaki are with the European Spallation Source ERIC, 22363 Lund, Sweden (e-mail: thomas.kittelmann@esss.se).

E. Klinkby and X. X. Cai are with the European Spallation Source ERIC, 22363 Lund, Sweden, and also with DTU Nutech, Technical University of Denmark, Ris $\varnothing$ Campus, 4000 Roskilde, Denmark.

C. P. Cooper-Jensen is with the European Spallation Source ERIC, 22363 Lund, Sweden, and also with the Department of Physics and Astronomy, Uppsala University, 75237 Uppsala, Sweden.

R. Hall-Wilton is with the European Spallation Source ERIC, 22363 Lund, Sweden, and also with the Department of Electronics Design, Mid-Sweden University, 85170 Sundsvall, Sweden.

Color versions of one or more of the figures in this paper are available online at http://ieeexplore.iee.org.

Digital Object Identifier 10.1109/TNS.2017.2695404

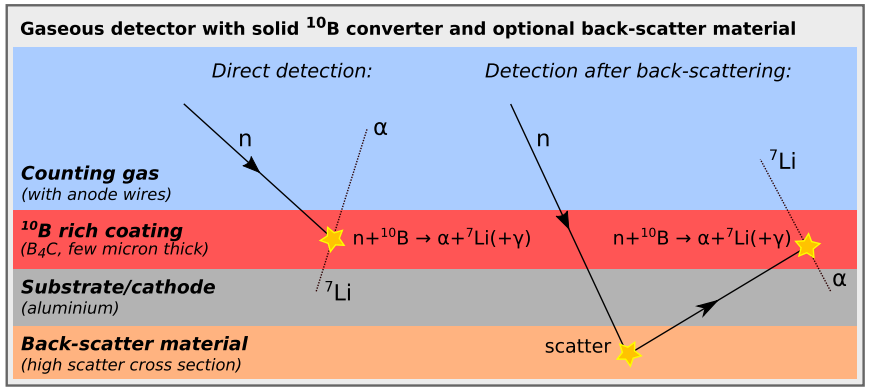

Fig. 1. Principle of a ${ }^{10} \mathrm{~B}$-based thin-film detector for thermal and cold neutrons.

${ }^{155,157} \mathrm{Gd}$, and ${ }^{235} \mathrm{U}$ have significant cross sections for such conversions, and detector systems must therefore contain such materials as well as incorporate capabilities to detect the resulting secondaries. The dominant detector choice has historically been gaseous ${ }^{3} \mathrm{He}$ detectors, based on the high cross-sectional process $\mathrm{n}+{ }^{3} \mathrm{He} \rightarrow{ }^{3} \mathrm{H}+p$. However, due to increased demand and decreased supply, ${ }^{3} \mathrm{He}$ will be unavailable in the future for all but the smallest detectors [7], [8]. Gaseous detectors, typically multiwire proportional chambers [9]-[12], are used to measure the energetic charged particles released in this conversion process. Thus, an extensive international research and development program is currently ongoing [13], [14] in order to develop efficient and cost-effective detectors based on other isotopes, and it is expected that detectors for the ESS will be based, as determined by the requirements of a given instrument, on technologies ranging from choices such as ${ }^{6} \mathrm{Li}$-containing scintillator detectors [6], [15]-[20] and Gd-based gas electron multiplier detectors [21] to gaseous detectors lined with thin films containing ${ }^{10} \mathrm{~B}$ [5].

There is a class of instruments [22] where detectors are required to, at a reasonable cost, cover large areas with detectors offering high rate capabilities, modest resolutions, and modest to high efficiencies. For these instruments, the most promising candidate is gaseous detectors surrounded by solid converters in the form of thin films of ${ }^{10} \mathrm{~B}$-enriched boron carbide [23], [24]. The basic principle of a successful direct detection event in the latter is illustrated on the left of Fig. 1: after conversion $\mathrm{n}+{ }^{10} \mathrm{~B} \rightarrow \alpha+\mathrm{Li}^{7}(+\gamma)$, one of the released ions travels into the instrumented counting gas where it can be detected like any energetic charged particle would. Typically, the instrumentation would include anode wires, with the boron carbide itself acting as the cathode, and such a detector therefore has the inherent high rate capability of any such a gaseous detector in proportional mode. 
Furthermore, due to the higher amount of energy released in the conversion reaction involving ${ }^{10} \mathrm{~B}(2.3 \mathrm{MeV})$ compared with the corresponding ${ }^{3} \mathrm{He}$ reaction $(0.77 \mathrm{MeV})$ and the implied large signals, the technology offers the possibility for very high suppression of gamma backgrounds [25], which can otherwise be a problem for neutron instruments. Additionally, it is a relatively cheap technology, allowing for large detector coverage when needed. However, the main limitation of the method is that high conversion efficiencies $(>50 \%)$ require the neutron to traverse several tens of micrometers of the converter, whereas the resulting $\alpha$ and $\mathrm{Li}$ ions only have a reach of a few micrometers in solid materials. Thus, to obtain higher detection efficiencies, one will typically either place many independent layers of gas-facing converters in the neutron path or try to arrange the geometry, so as to keep the angle of incidence of the neutron on the converter as high as possible, or a combination of the two (noting that we here adopt the convention that a $0^{\circ}$ incidence angle corresponds to normal and $90^{\circ}$ to grazing incidence). Detailed analytical calculations of detection efficiency, depending on inclination and converter thickness, exist [26]. However, such solutions come at a penalty of increased complexity and cost, and this paper instead investigates the performance of an alternative approach in which detection efficiency is increased by the addition of a strongly scattering material at the back of the detector.

\section{RECOVERING UNCONVERTED NEUTRONS THROUGH BACKSCATTERING}

The use of backscattering materials with the aim to improve performance by neutron reflection is by no means new and has seen a great deal of applications wherever moderating materials are used, e.g., in dosimetry [27]-[29] in the context of neutron albedo and in reflectors used in reactors [30] or radiation detectors [31], [32]. Inspired by these neutronspecific applications, this paper is attempting to illustrate what new improvements an established technique can bring to the thermal neutron detection field for neutron scattering applications.

The principle of the method investigated in this paper is straightforward, as illustrated on the right side of Fig. 1. Placing a material with a high backscattering cross section behind a neutron detector will increase detection efficiency, since a neutron that did not convert in the detector in the first place will have a nonzero probability of scattering back to the detector and converting the second time around, thus recovering events, which would otherwise go undetected.

The downsides to adding such a scattering material would be expected to be twofold: not only the events thus recovered would suffer from degraded position resolution as well as a systematic positive shift in detection time, but also one would potentially have to worry about backscattered neutrons escaping the detector through the front. The latter could potentially be a problem if the instrument in question features detectors at opposite sides of a sample in which neutrons scatter before detection. Collimators in front of the detectors and shielding between detector sections would often be needed in any case, and can be expected to significantly lower the negative impact of this effect. Additionally, if the chosen backscattering material primarily scatters incoherently, one would avoid unduly adding new features to the detected distributions, coming from coherent scattering. For any given instrument, one would need to carefully analyze the exact implementation details of proposed designs in order to ensure that all such effects would be understood, preferably through a combination of tests on prototypes and simulations of the complete envisioned setup.

The requirement of high cross sections for incoherent scattering of thermal neutrons suggests that a hydrogen-rich moderator-like material would be a good candidate backscattering material. From an operational and a costing point of view, the most suitable candidate would a priori seem to be a plastic, such as the widely used and studied moderator and shielding material, polyethylene (PE), which is therefore the material we will focus on in the present investigations.

Regarding the placement of the backscattering material, it should ideally be immediately after or as close as possible to the last conversion material in the detector. This is so as to reduce the additional time shift and spatial displacement of the backscattered neutrons before a potential conversion. Furthermore, it is important that any material between the last conversion material and the backscattering material will have a very low probability of neutron absorption. Finally, it is also clear that multilayered detectors in which the depth of interaction is used to estimate the total time of flight and hence the energy of the neutron would not be suitable to be extended with a backscattering material.

Given the above considerations, the present investigations will focus on the performance impact of adding a layer of PE behind a single-layered ${ }^{10} \mathrm{~B}$-thin-film-based gaseous detector, as is illustrated in Fig. 1. Although different setups could be envisioned, the detector will for simplicity be taken to be implemented as in Fig. 1, with an aluminum substrate placed at the backside of the gas only. The substrate, which in actual detectors is used to provide necessary mechanical support and electrical potential, is coated with a thin layer of the conversion material and the PE is located at its back. A multilayer setup in an instrument that does not rely on time-of-flight information would also be possible, but in general multilayer setups already come with high detection efficiencies and price tags, and the presently discussed method is more likely suitable for the opposite scenarios.

\section{Simulations}

The exact impact on the instrument and detector performance of adding a backscattering material to a detector at a neutron scattering instrument will depend in detail on many factors. The first factor includes the details of the detector itself, including its technology, layout, and how the backscattering material is integrated. Other factors include the distribution with which neutrons are delivered at the sample position, the typical samples to be investigated, and the typical analyses that will subsequently be carried out with the recorded data. Despite this high level of complexity, it is nonetheless possible to evaluate the feasibility of the backscattering concept by considering a specific and simple, 
TABLE I

Summary of MATERIALS AND Dimensions USED IN THE Simulation

\begin{tabular}{lllll}
\hline Layer & Material & Isotopic composition & Density & Thickness \\
\hline Counting gas & $70 \%-30 \% \mathrm{Ar}-\mathrm{CO}_{2}$ (by mass). & Natural & $1.833 \mathrm{mg}_{\mathrm{cm}}^{3}$ & $10 \mathrm{~mm}^{2}$ \\
Converter & Boron carbide, $\mathrm{B}_{4} \mathrm{C}$ & $98 \%-2 \% \mathrm{~B}^{10}-\mathrm{B}^{11}$ (by mole), C natural & $2.378 \mathrm{~g} / \mathrm{cm}^{3}$ & $0.1-5 \mu \mathrm{m}$ \\
Substrate & Aluminium & Natural & $2.699 \mathrm{~g} / \mathrm{cm}^{3}$ & $0.5 \mathrm{~mm}$ \\
Back-scatter & Polyethylene, $\mathrm{C}_{2} \mathrm{H}_{4}$ & Natural & $0.940 \mathrm{~g} / \mathrm{cm}^{3}$ & $0-50 \mathrm{~mm}$ \\
\hline
\end{tabular}

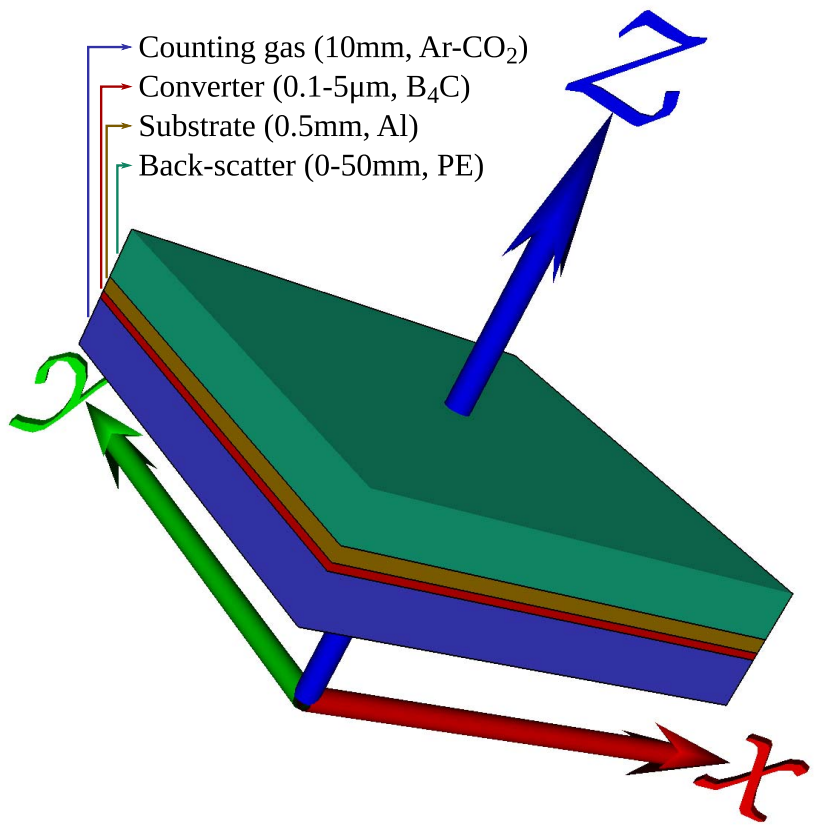

Fig. 2. Visualization of the geometry used for the simulations. Neutrons are shot in the direction of the $z$-axis (blue arrow), which in the shown configuration are at normal incidence to the detector plane, and encounter layers of counting gas (blue layer), converter (red layer), substrate (brown layer) and backscattering material (green layer). For clarity of visualization purposes, the converter and substrate thicknesses have been blown up and the transverse extent of the plane reduced.

yet reasonably realistic setup. The present investigations will therefore consist of realistic Monte Carlo simulations of a setup exactly like the one depicted in Fig. 1, in which layers of counting gas, converter, substrate, and possibly a backscattering material will be placed in the path of incoming neutrons. The layers will be assumed to be very large in the transverse dimensions.

\section{A. Setup Details and Verification}

The simulations are implemented and carried out using the Geant4 [33]-[35] based framework described in [36], and the simulated geometry is shown in Fig. 2. For reference, the present investigations are implemented in Geant4 version 10.0 .3 with a $70 \%-30 \%$ (by-mass) counting gas mixture of $\mathrm{Ar}-\mathrm{CO}_{2}$, a boron-carbide converter enriched to $98 \%{ }^{10} \mathrm{~B}$, and the gas and substrate plane thicknesses of 10 and $0.5 \mathrm{~mm}$, respectively. These values represent typical parameters found in planned ${ }^{10} \mathrm{~B}$-lined neutron detectors [37]-[41], and with the exception of the ${ }^{10} \mathrm{~B}$ enrichment level, the results of the present analysis are not believed to be overly sensitive to them in typical setups of nonextreme inclination with respect to the incident neutrons. This is usually guaranteed by design, because the physical dimensions and materials will realistically be chosen by detector developers so that thermal neutrons rarely interact only inside the volumes of the counting gas or substrate. Noting that the transverse dimensions of the layers are set so large as to be essentially infinite for the purposes here, the remaining parameters are summarized in Table I.

The previous studies [42] and [43], which also included comparisons with measurements, have shown that Geant4 with the QGSP_BIC_HP physics list is able to adequately capture the physics of neutron absorption in enriched boron carbide and the subsequent journey of the released ions into the counting gas. It was also shown that a simple threshold on the amount of energy deposited therein suffices to accurately emulate actual detection efficiencies in a real detector equipped with anode wire planes in the counting gas. This is of course not surprising, as one would first of all expect Geant4 to describe absorption of thermal and colder neutrons well, given that the relevant cross sections are given by a simple $\sim 1 / v_{n}$ law. The second is that a good description of energy loss by charged particles in matter is an essential feature of Geant4, used as it is to routinely model energy depositions in a broad range of applications. Thus, in the context of the simulations, we shall define a neutron as being detected when its simulation leads to a total of at least $150 \mathrm{keV}$ of energy being deposited in the counting gas volume (with detection position and time inferred from the coordinates of the particles performing the depositions).

However, materials in Geant 4 are usually described under a free gas assumption, with no information concerning interatomic chemical bindings. This means that potentially important features such as Bragg diffraction in a polycrystalline material like aluminum and thermal scattering (TS) on energy levels in hydrogen bonding in PE are a priori missing. For the former issue, the setup is therefore augmented to use the NXSG4 extension [44], to ensure an accurate description of the aluminum used in the substrate. For the latter issue, it is fortunate that PE, seeing significant use for neutron moderation and shielding, is one of the selected few materials for which detailed TS cross-sectional data at various temperatures exist, although different codes and data versions might provide slightly different results. Here, we will compare a model shipped with Geant 4 itself, due to work by T. Koi, with a custom in-house model to evaluate the JEFF-3.2 ACE formatted files for the TS Law [45], as well as, for reference, with the output from simulations carried 


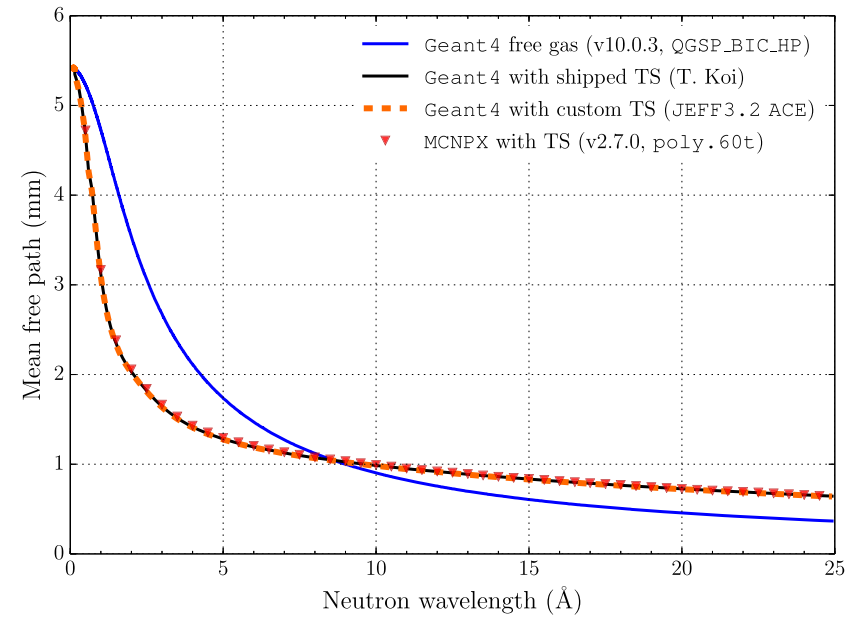

Fig. 3. Mean free path of neutrons in PE in Geant 4 and MCNPX, as a function of neutron wavelength and for different physics models. The methods for extracting these values are discussed in the text.

out with MCNPX version 2.7.0 [46] using the poly.60t thermal cross sections derived from ENDF6.5 dynamic structure factors using NJOY [47]. The comparison with MCNPX is of particular importance here, as it is typically relied on for simulations of TS of neutrons in PE for shielding and moderation purposes, and has been validated against measurements [48], [49]. Choosing pragmatically a material temperature of $293.6 \mathrm{~K}$, which is available for $\mathrm{PE}$ in all three implementations, and comparing also with the base free gas treatment in QGSP_BIC_HP, the resulting mean free path lengths of neutron interactions in PE are shown in Fig. 3 (note the relation between neutron wavelength and energy: $\left.E[\mathrm{eV}]=0.0818 /(\lambda[\AA ̊])^{2}\right)$. Note that for reliability, the values in Fig. 3 were extracted at runtime rather than second guessed from data files. For Geant4, this was done via a custom hook querying the physics processes GetMeanFreePath (..) methods. For MCNPX, the values were determined through direct simulations of neutrons impinging on a very thin plane of $\mathrm{PE}$ and the resulting statistical errors are smaller than the plot markers. The importance for our analysis of using specific TS data for PE, rather than just the base free gas model is clear, as the resulting mean free path length for neutrons scattering in PE is affected with as much as a factor of 2.

As a further comparison, Fig. 4 shows the simulated transmission spectrum of $2.5 \AA$ neutrons moderated in a 50-mm-thick slab of PE. For the purposes of the present investigations, the three curves with specific TS models are compatible and different from the free gas model. However, as the present investigations involve backscattering in potentially very thin layers of $\mathrm{PE}$, it is interesting to compare the backscattered spectrum after a single interaction. Thus, Fig. 5 shows a comparison of the backscattered spectrum in a $100-\mu \mathrm{m}$ PE slab, of which two interesting observations can be made. First of all, the three thermal scattering models all include an incoherent elastic peak at the energy of the incoming neutron $(2.5 \AA-13.1 \mathrm{meV})$, whereas this is absent in the free gas model. Second, while it is once again clear that

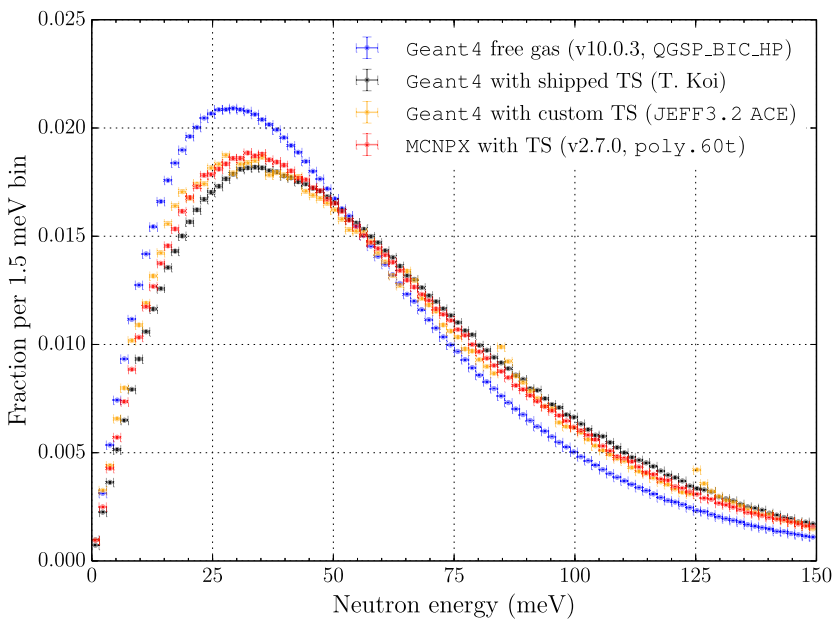

Fig. 4. Simulated transmission spectrum for $2.5 \AA$ (13.1 meV) neutrons through $50 \mathrm{~mm} \mathrm{PE}$ in both Geant 4 with different physics models and MCNPX.

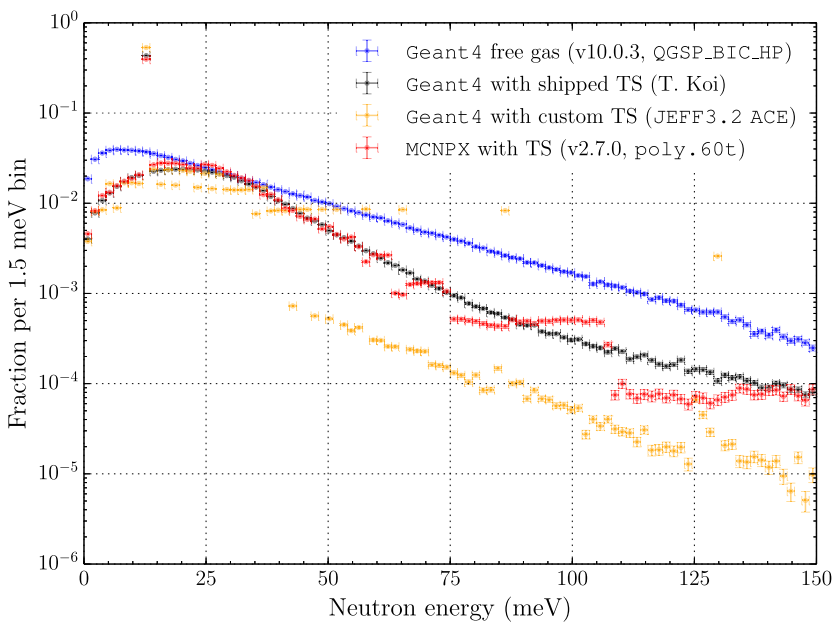

Fig. 5. Simulated backscattered spectrum for $2.5 \AA(13.1 \mathrm{meV})$ neutrons on $100-\mu \mathrm{m}$ PE in both Geant 4 with different physics models and MCNPX.

the general shapes of the distributions for the three TS models follow the same rough shape, it is only the one shipped with Geant 4 which does not exhibit artifacts due to the discrete parameterizations of the TS data, and for that reason, this is the model chosen for the investigations in the following sections.

\section{B. Results}

As an illustration, simulated trajectories of particles resulting from firing 50 neutrons into our setup are visualized in Fig. 6: neutrons arriving from below travel through the counting gas and either convert in the enriched boron carbide immediately, releasing charged ions into the gas, or pass onward through the substrate and into the PE where complex trajectories follow as a result of the multiple scattering interactions. Ultimately, those neutrons pass through to the backside of the PE, get absorbed in processes releasing photons, or return back to the substrate and converter, where some are absorbed the second time around as hoped, again releasing ions into the counting gas. 


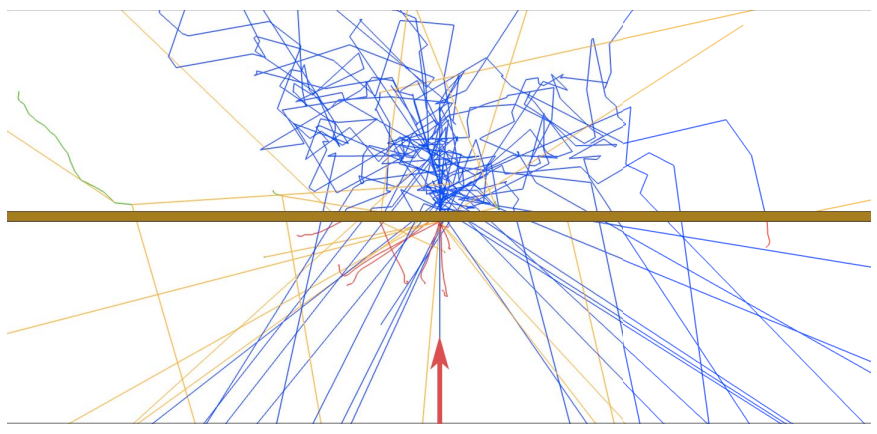

Fig. 6. Simulated trajectories of $501.8 \AA \AA$ neutrons (blue lines) starting from the center of the bottom edge of the figure (indicated by a red arrow) and traveling upward through layers of counting gas, $2.5 \mu \mathrm{m}$ of enriched boron carbide, $0.5 \mathrm{~mm}$ of the aluminum substrate (brown layer), and finally $10 \mathrm{~mm}$ of PE. As a result of neutron absorption, secondary particles are released in various locations: photons (orange lines), electrons (green line) and $\alpha$ or $\mathrm{Li}^{7}$ ions (red lines).

The key issues to be investigated with the simulations are on one hand what positive impact placing different amounts of PE will have on detection efficiency and on the other hand what the associated negative impacts on time and position resolution will be, and to what extent those can be mitigated. The answers can be expected to depend also on the angle of incidence of the neutron on the detection plane, the neutron energy, and the chosen thickness of the converter coating. To answer these questions, large numbers of neutrons were simulated for a variety of configurations and the results subsequently carefully analyzed in order to produce the plots in this section. For reference, a total of $3.5 \times 10^{10}$ neutron events were simulated, using between $4.0 \times 10^{6}$ and $2.0 \times 10^{8}$ for each given configuration, manually chosen so as to achieve low statistical fluctuations in all plots.

First, simulated detection efficiencies at normal incidence as a function of converter thickness are shown in Fig. 7 for various amounts of PE and for both thermal (1.8 $)$ and cold $(7.0 \AA ̊)$ incident neutron energies. As expected, detection efficiencies for colder neutrons are higher, but otherwise the qualitative features of the curves are similar to those at thermal energies. For simplicity, the remainder of our investigations will therefore focus on neutrons with a wavelength of $1.8 \AA$. The next thing to note is that the curves without PE grow monotonically with converter thickness, but effectively saturate at the final plateau already around $2.5 \mu \mathrm{m}$, which is the effective range in the converter of the ions released in the conversion. These converter thicknesses compare well with the expectation from the analytical calculations [26], lending further credence to the simulation. On the other hand, when PE is added, the curves exhibit a maximum around $2.1-2.5 \mu \mathrm{m}$, which is expected since conversions at ever deeper locations are increasingly unlikely to contribute positively with direct detection events, and will merely act as unwanted inactive shielding in the path of neutrons being backscattered by the PE. Assuming detector developers to always adopt the most favorable converter thickness for their chosen setup, its value should ideally be optimized individually for each configuration considered in a given comparison of multiple such setups. Fortunately, however, it appears that all curves in Fig. 7 are

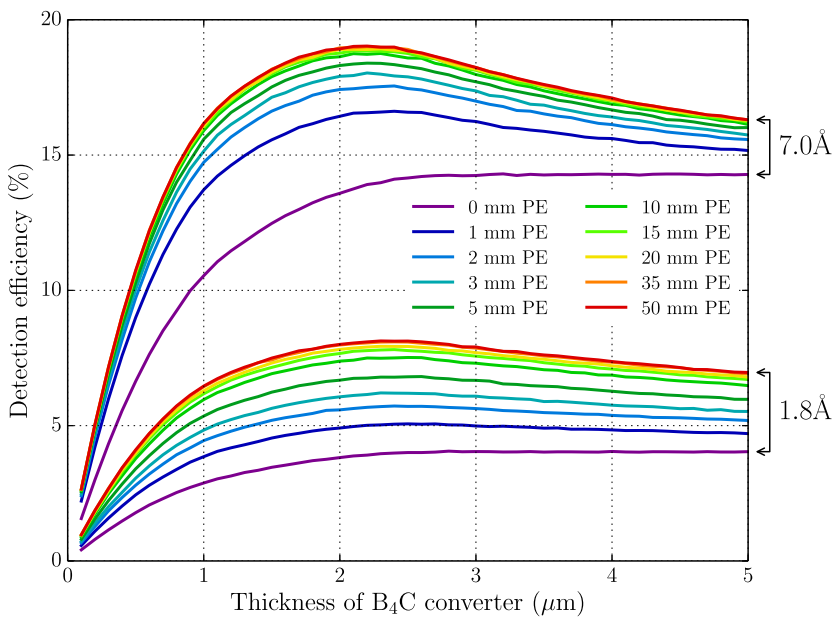

Fig. 7. Simulated detection efficiency for neutrons at normal incidence as a function of converter thickness, for various neutron energies and amounts of PE.

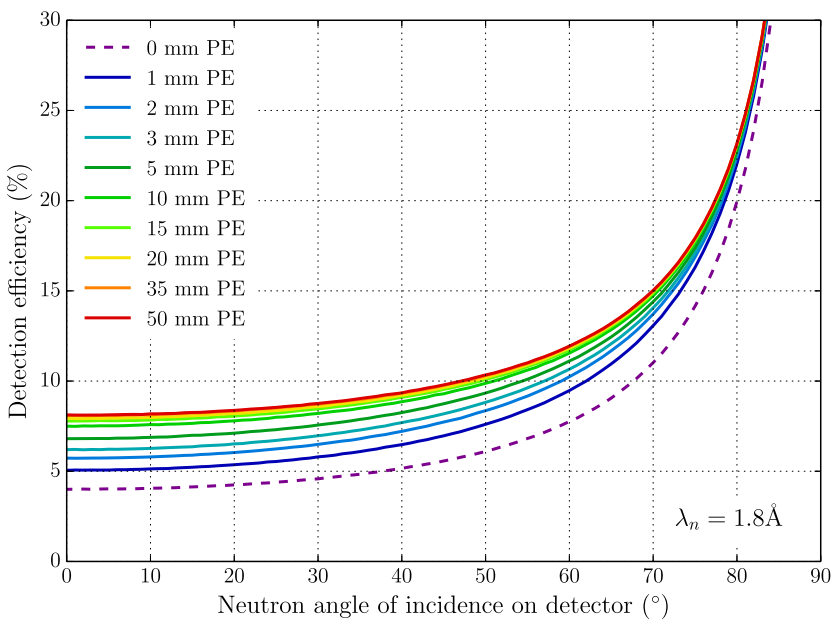

Fig. 8. Simulated detection efficiency for various amounts of PE as a function of the incidence angle between the incoming neutron and the detector plane.

reasonably close to their maximum value when the converter thickness is set to $2.5 \mu \mathrm{m}$, meaning that this value can be used for all setups throughout the remainder of the present investigations without unduly biasing the comparisons. Finally, it appears as could be expected that detection efficiencies grow only with the amount of PE added, but at an ever decreasing rate-the largest gain coming from the first few millimeters added.

Next, Fig. 8 shows the detection efficiency as a function of incidence angle of the neutron on the detector planes. As expected, the efficiency curves all increase sharply as the incoming neutron tends toward grazing incidence. However, as is seen more clearly in Fig. 9, the relative gain in detection efficiency from adding the PE decreases at higher incidence angles. This is understood knowing that the backscattering from the PE is incoherent and thus effectively isotropic: at low incidence angles, a random backscattered neutron is likely to hit the converter at a higher incidence than during the 


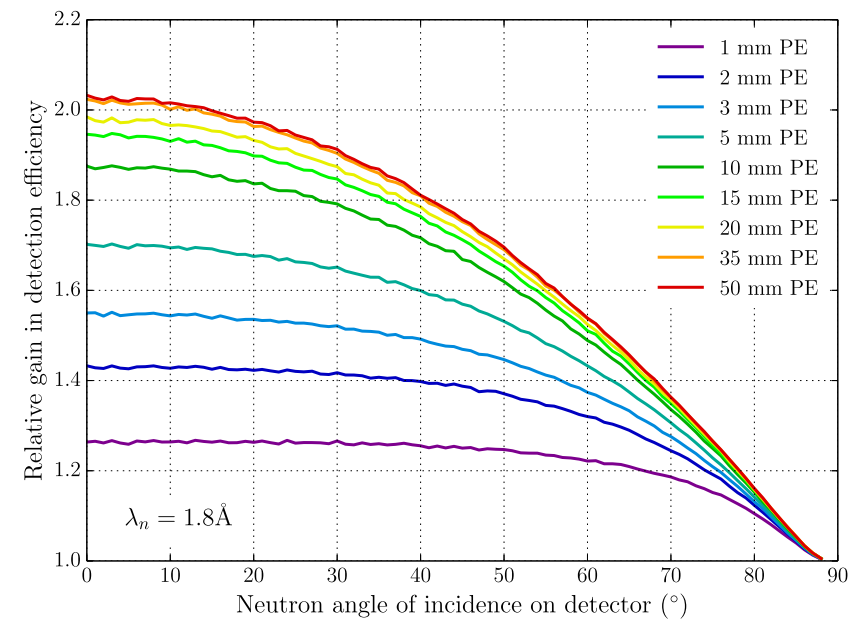

Fig. 9. Simulated relative gain in detection efficiency for various amounts of PE as a function of the incidence angle between the incoming neutron and the detector plane.

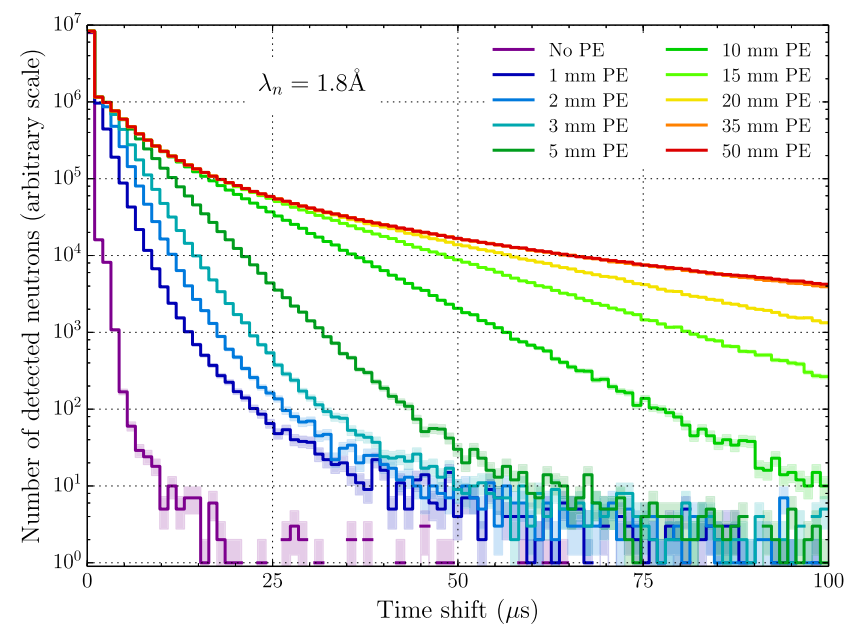

Fig. 10. Simulated distribution of shift in detection time for various amounts of PE.

initial traversal, whereas at high incidence angles, the situation is reversed. Thus, while the result of Fig. 9 is promising very tangible improvements at low incidence angles, it is also making it clear that the concept is not suitable for detectors that are to be operated at higher incidence angles. For that reason, the rest of the present investigations will focus on neutrons with low incidence angle (perpendicular to the surface).

Turning to the possible downsides, Fig. 10 shows the distribution of simulated detection time for various amounts of PE, counting from the moment the neutron first enters the converter and until the time when energy exceeding the threshold is deposited in the countring gas, usually by the released ions. It is clear that addition of PE behind the detector leads to increasing tails toward larger times, but in order to quantify the effects, it is arguably more useful to look instead at the curves showing the fraction of neutrons with detection time shifts above a given threshold shown in Fig. 11. For instance, one can learn that if a specific detector has

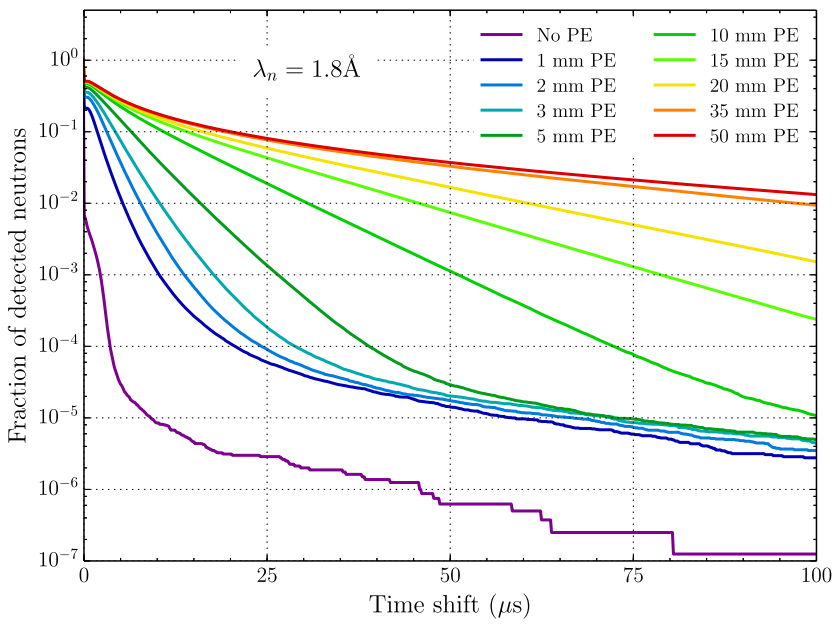

Fig. 11. Simulated fraction of neutrons above a given shift in detection time for various amounts of PE.

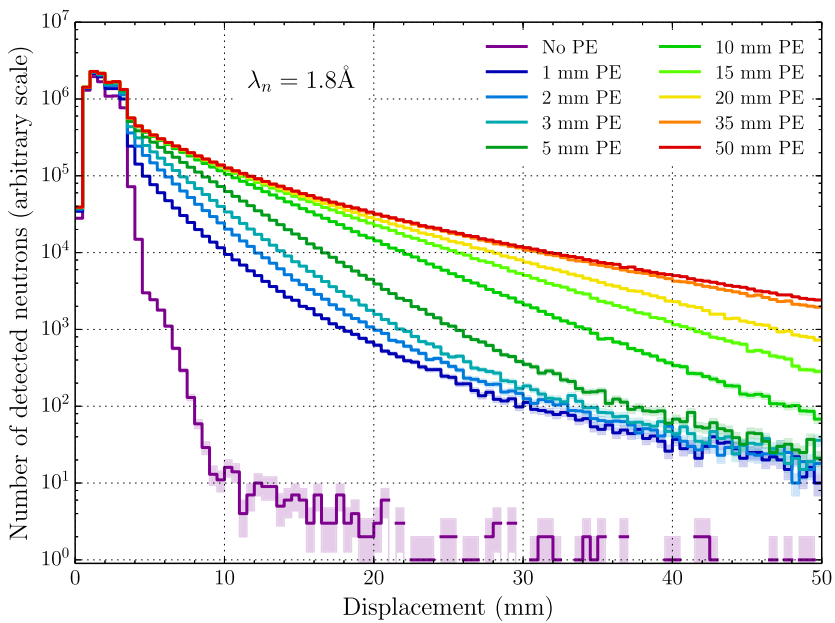

Fig. 12. Simulated distribution of displacement in detection location for various amounts of $\mathrm{PE}$.

a requirement that all except $10^{-3}$ of the detected neutrons must be detected with a time shift less than $50 \mu \mathrm{s}$, one should not add more than $\sim 10 \mathrm{~mm}$ of backscattering PE. Fortunately, even a $100-\mu$ s resolution at the $10^{-2}$ level would be adequate for many neutron instruments [5].

Next, Figs. 12 and 13 show the corresponding distributions of the spatial displacement of the detection location, given by the location of energy depositions in the counting gas, relative to the position where the neutron first enters the converter. Again, we can readily read the performance metrics from the second of the figures: if one requires all except $10^{-2}$ of neutrons to be detected with a displacement less than $20 \mathrm{~mm}$, no more than $10 \mathrm{~mm}$ of $\mathrm{PE}$ should be added behind the detector.

\section{Possible Mitigation Strategies}

The results so far, summarized in Figs. 9, 11 and 13, indicate that the presented method has the potential to provide substantial gains in detection efficiencies, but with potentially significant associated adverse effects on both temporal and 


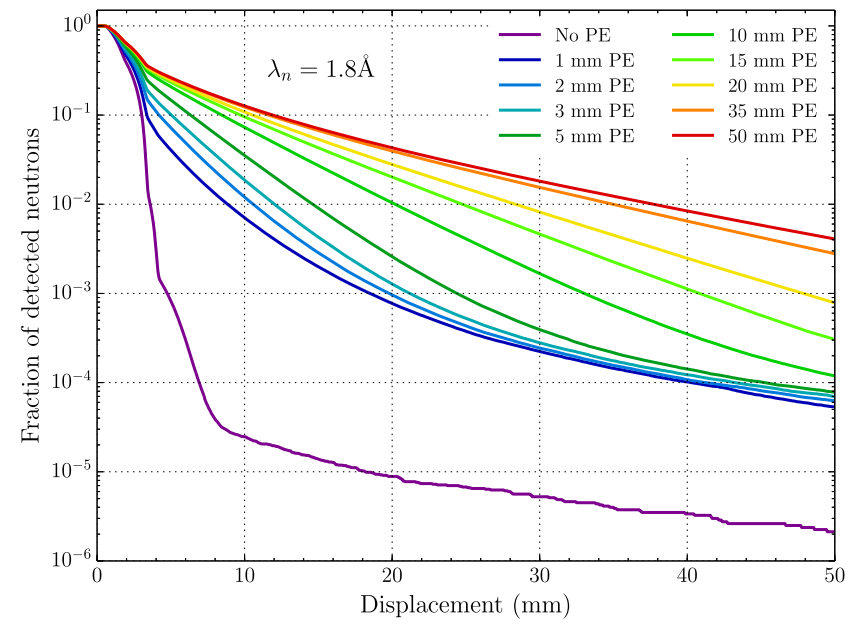

Fig. 13. Simulated fraction of neutrons having a displacement in detection location above a given threshold, for various amounts of PE.

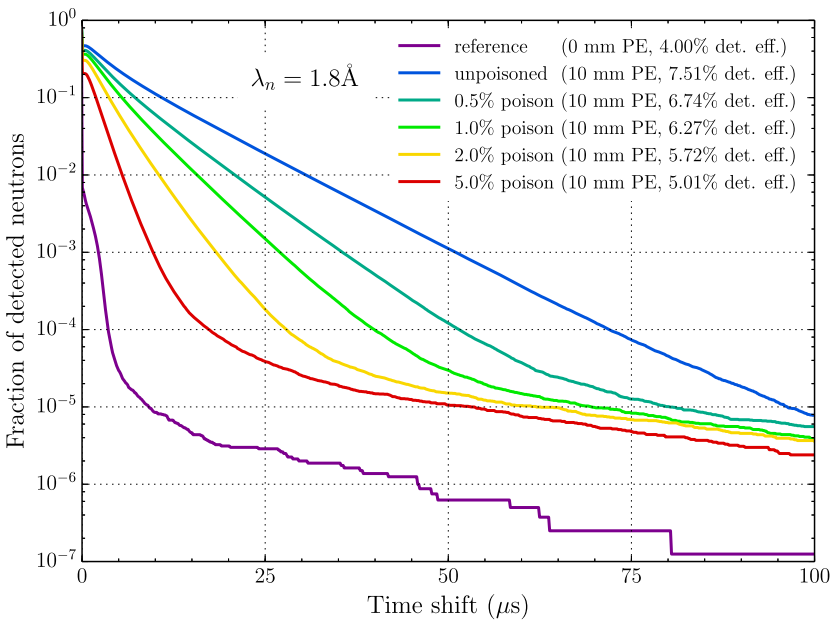

Fig. 14. Simulated fraction of neutrons above a given shift in detection time for no PE as well as 10-mm PE with various levels of poisoning.

spatial detection resolutions. In this section, we will briefly investigate two strategies for reducing the impact of these adverse effects. In essence, both will try to eliminate some or all unfavorable trajectories in the $\mathrm{PE}$, while hopefully retaining a large fraction of the beneficial ones. Ignoring events with neutrons either absorbed inside or transmitted through (to an absorbing backside most likely) the PE, favorable trajectories are those where the neutron is backscattered out of the front of the PE with as small a distortion of distance and time as possible. Unfavorable trajectories are on the other hand those where the neutron either spends a long time inside the PE (impacting temporal detection) or travels a long transversal distance inside the PE (impacting spatial detection).

To eliminate the first type of unfavorable trajectories, one might consider "poisoning" the PE, by contaminating it with a small fraction of atoms with high cross section for neutron absorption. Done right, this should ideally result in a large fraction of those neutrons spending a long time inside the PE being absorbed, with only little impact on

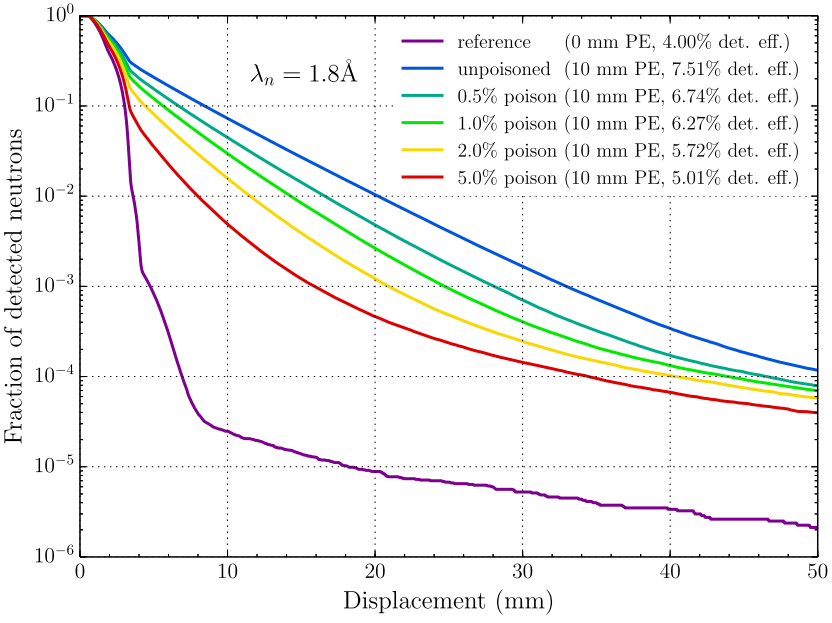

Fig. 15. Simulated fraction of neutrons having a displacement in detection location above a given threshold, for no PE as well as 10-mm PE with various levels of poisoning.

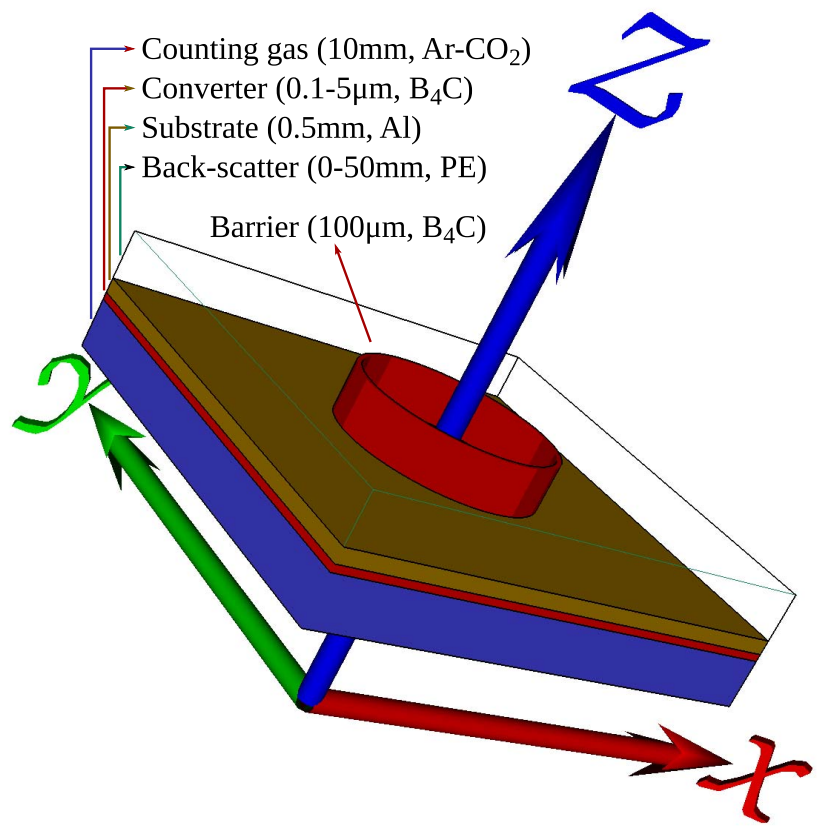

Fig. 16. Visualization of the geometry as in Fig. 2, but with the backscattering material opened up to show how a simple cylindrical barrier has been added inside, to contain the neutrons in a well-defined region. As in Fig. 2, neutrons are shot in the direction of the $z$-axis (here shown at normal incidence to the layers), encountering layers of counting gas, the converter, the substrate, and the backscattering material in that order. For clarity of visualization purposes, the converter and substrate thicknesses have been blown up and the transverse extent of the plane reduced.

neutrons promptly backscattered. Any choice of element typically used as a converter in thermal neutron shielding could be considered for such poisoning purposes, such as cadmium, gadolinium, or boron. Of these, boron is the most straightforward choice for the present purposes, as it is cheap, neither hazardous nor toxic, and a low number of gammas are emitted during neutron conversion. To quantify the potential of poisoning, Fig. 14 shows the simulated effect in a setup with 10-mm PE. For example, adding $0.5 \%$ (by-volume) of 


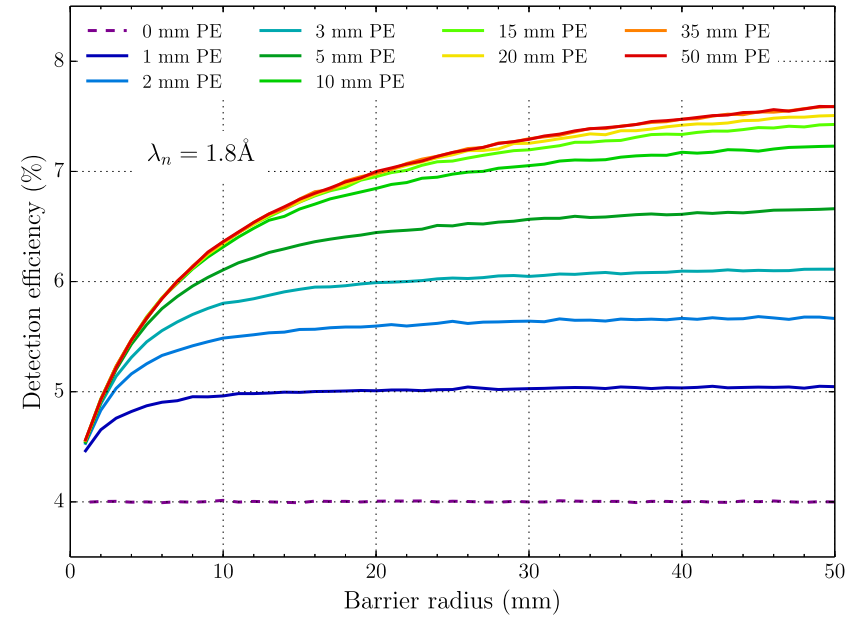

Fig. 17. Simulated detection efficiency for various amounts of PE in the presence of a cylindrical barrier as a function of the barrier radius.

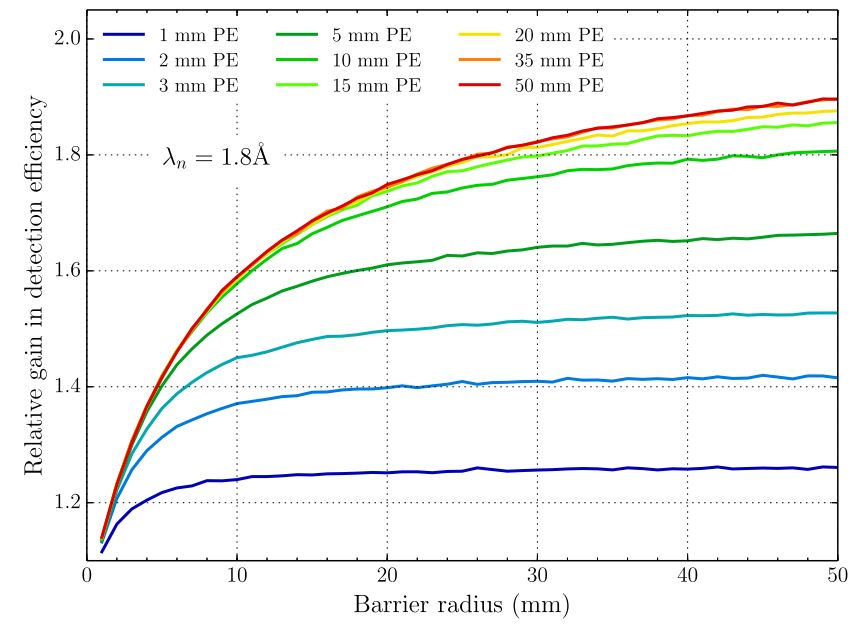

Fig. 18. Simulated relative gain in detection efficiency for various amounts of $\mathrm{PE}$ in the presence of a cylindrical barrier as a function of the barrier radius.

natural boron reduces the fraction of neutrons detected above $50 \mu$ s by an order of magnitude, while reducing the detection efficiency only from $7.51 \%$ ( $88 \%$ above the reference $4 \%$ ) to $6.74 \%$ (69\% above the reference $4 \%)$. Poisoning is thus a potentially very potent method, if one is concerned with the detection time resolution. On the other hand, Fig. 15 shows that the impact on the spatial resolution is somewhat smaller, which is as expected since the unfavorable trajectories traveling a long transversal distance in PE are not necessarily always spending a very long time in it.

Fortunately, tails in the spatial resolution are easily handled in the context of real detectors, which are typically segmented according to their required granularities, charge collection, and readout schemes. Thus, by segmenting the PE as well, separating different detector cells by an appropriate absorbing material, one can be certain that a neutron entering the PE from within a given cell will only be able to be backscattered to the

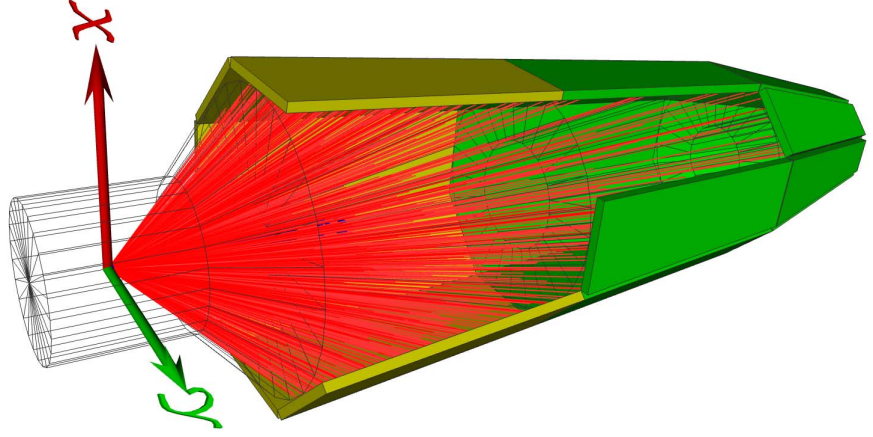

Fig. 19. View of the LoKI "tapered" detector geometry as defined in the Geant4 simulation. A Christmas-tree-shaped vacuum tank is equipped with three banks of ${ }^{10} \mathrm{~B}$-based detectors. The detector bank under investigation is marked by yellow volumes and neutrons stemming from the sample position are shown by red lines.

same detection cell. The question then instead becomes one of how much the detection efficiency gain due to the PE will be reduced due to neutrons meeting the absorber between PE cells. This will again depend on the exact detector design, but to quantify the effect and the dependency on the size of detection cells, simulations were carried out with a cylindrical barrier of $100-\mu \mathrm{m}$ enriched boron carbide placed in the PE, as shown in Fig. 16, and detection efficiencies were simulated for neutrons at normal incidence generated uniformly over the corresponding circular detection cell. The result is shown in Figs. 17 and 18: for very small detection cells, the absorption in the barrier almost completely eliminates the gain from adding the PE, but for a realistic barrier radius of $20 \mathrm{~mm}$, $10 \mathrm{~mm}$ of PE will still provide a relative gain in detection efficiency of approximately $70 \%$.

\section{LOKI "TAPERED" Detector GeOMETRY: A USE CASE}

The methodology presented in this paper is developed as an additional tool for the design of neutron detectors in neutron scattering applications. As an example of a possible use case, based on taking advantage of the detailed scientific requirements of the application, the effect has been evaluated for a suggested detector geometry for the LoKI [50] smallangle neutron scattering (SANS) instrument [51].

The example chosen is that of a novel and futuristic detector geometry, whose concept is described in [52] and [53] and shown in Fig. 19. A Christmas-tree-like vacuum tank is lined with three banks of ${ }^{10} \mathrm{~B}$-based detectors, offering extensive polar angle $(\theta)$ coverage, in order to satisfy one of the major scientific requirements of the LoKI instrument, and broad angular coverage of scattered neutrons.

The focus of the case study is the octagonal detector array closest to the sample, covering an approximate $\theta$ range between $20^{\circ}$ and $45^{\circ}\left(0^{\circ}\right.$ is the incident beam direction on the $z$-axis). The interior of the detector contains a single boron carbide $\left({ }^{10} \mathrm{~B}_{4} \mathrm{C}\right)$ converter layer on an aluminum substrate with the possibility to add a PE layer behind the latter. The geometrical arrangement of the detector materials is identical to the one of Fig. 2, with a boron carbide layer of $2.5 \mu \mathrm{m}$ thickness sitting on an aluminum substrate of $0.5 \mathrm{~mm}$. 


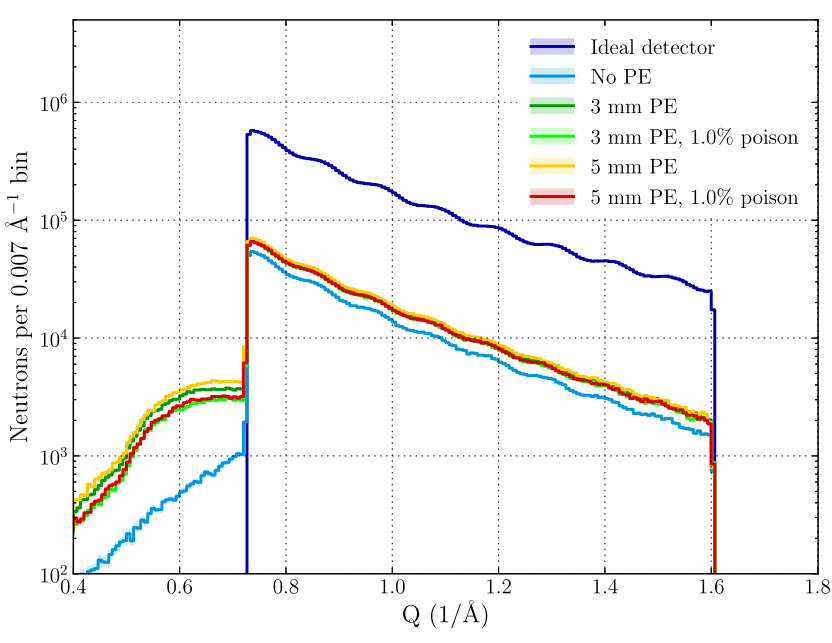

Fig. 20. $Q$ distribution in blue represents a typical SANS sample containing spherical structures with a radius of $500 \AA$ and corresponds to what an ideal detector would detect. The reconstructed $Q$ distributions from detected neutrons are represented by the rest of the colors for various conditions. The tails of the distributions are the result of the neutrons scattering inside the large geometry until their conversion. The statistical errors of the simulation are too small to discern.

As a figure of merit for the performance improvement that the $\mathrm{PE}$ addition can bring, the impact on the momentum transfer $Q$ is considered

$$
Q=\frac{4 \pi}{\lambda} \sin (\theta / 2)
$$

where $\theta$ is the polar angle with which the neutron is scattered on the sample and $\lambda$ is its initial wavelength. $Q$ is a typical variable considered in neutron scattering techniques and SANS in particular, as it contains information about the characteristic length scales of the sample under study and forms the basis for further data analysis. This implies that a decent $Q$ resolution is necessary for a high-quality analysis, which imposes certain requirements on the detector spatial and timing resolution.

Using the exact same simulation tools as described in the previous sections, the detector performance of such a geometry configuration can be studied in detail and the effects of a PE layer on the detection efficiency and the $Q$ resolution can be quantified. To this end, a typical $Q$ distribution has been used as the simulation input. It corresponds to a sample containing spherical structures with a $500 \AA$ radius, probed with an incoming neutron beam of $\lambda=3 \AA(9.09 \mathrm{meV})$. These neutron incident angles and low wavelength are one of the regions of scientific interest for the LoKI instrument. According to the results of this study, this is where the biggest improvement is anticipated.

From the simulation of the detector response, it is possible to derive information on the spatial and temporal locations of a detected neutron. This information is used to calculate the detected $Q_{\text {det }}$ value and compare it with the initial true one, $Q_{\text {true }}$. Having access to these two values, it is possible to calculate both the detection efficiency and the $Q$ resolution, thus convoluting all the factors that potentially smear the true $Q$ value in the detector response.

Fig. 20 demonstrates the aforementioned process. By choosing various combinations of PE thickness and boron poison as

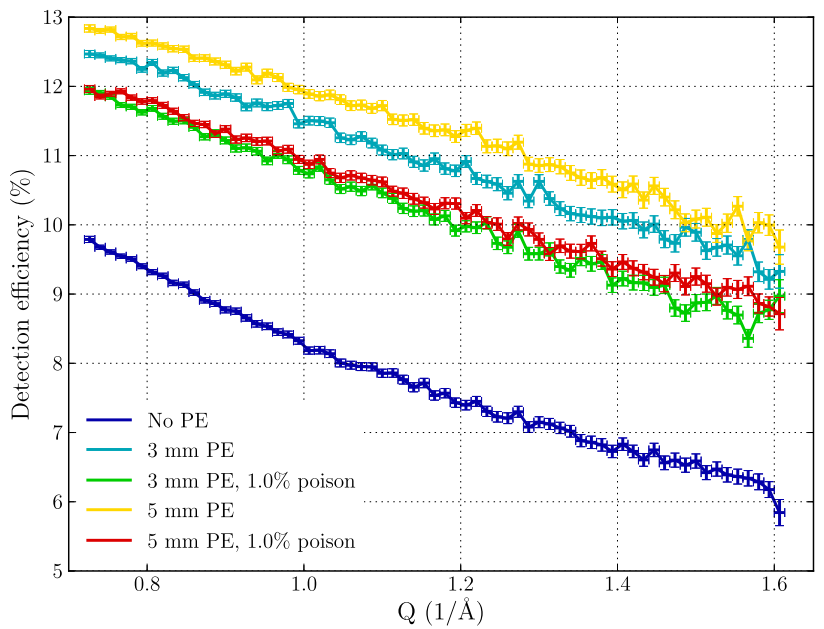

Fig. 21. Detection efficiency as a function of the scattering vector $Q$ for various combinations of PE thickness and boron poison content (by volume). The falling trend with increasing $Q$ is attributed to the respectively increasing neutron incident angle on the converter, which translates into lower detection efficiency.

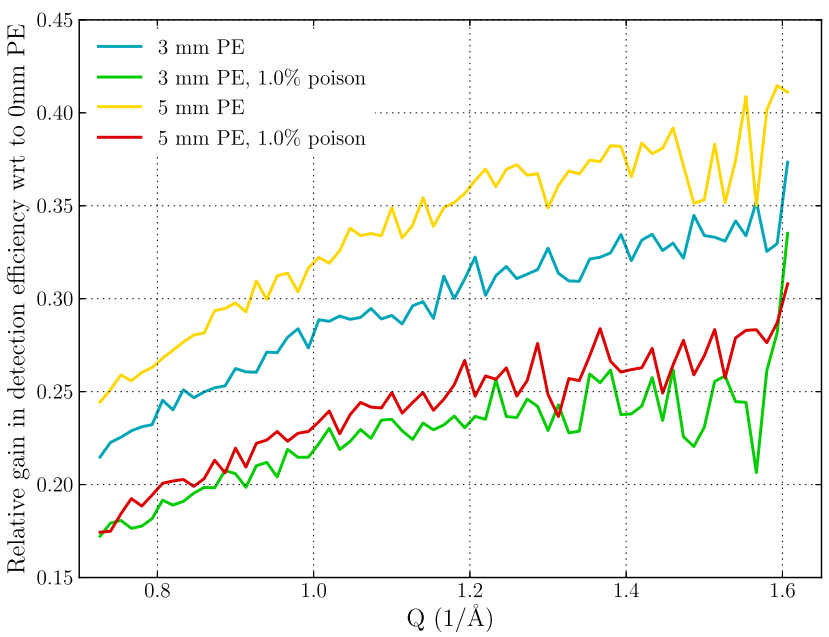

Fig. 22. Relative efficiency gain for various combinations of PE thickness and poison content, with respect to the absence of the backscattering layer.

a mitigation technique, according to the previous sections, it is possible to estimate the respective detection efficiency as a function of $Q$ (see Fig. 21) as well as the deviation of the reconstructed $Q$ value $Q_{\text {det }}$ from the initial true $Q$ value $Q_{\text {true }}$ (see Fig. 23) via the equation

$$
\delta Q / Q=\frac{Q_{\text {det }}-Q_{\text {true }}}{Q_{\text {true }}} .
$$

The addition of PE behind the detector substrate can impact the detection efficiency positively and the detection resolution negatively. This use case of detector design constitutes a typical optimization problem and the answer as to what combinations of PE thickness and absorbing poison are appropriate depends each time on the optimization priorities.

Fig. 22 quantifies the impact of the PE and the poison content on the detection efficiency as a function of $Q$. As expected, the detection efficiency increases with the PE thickness. 


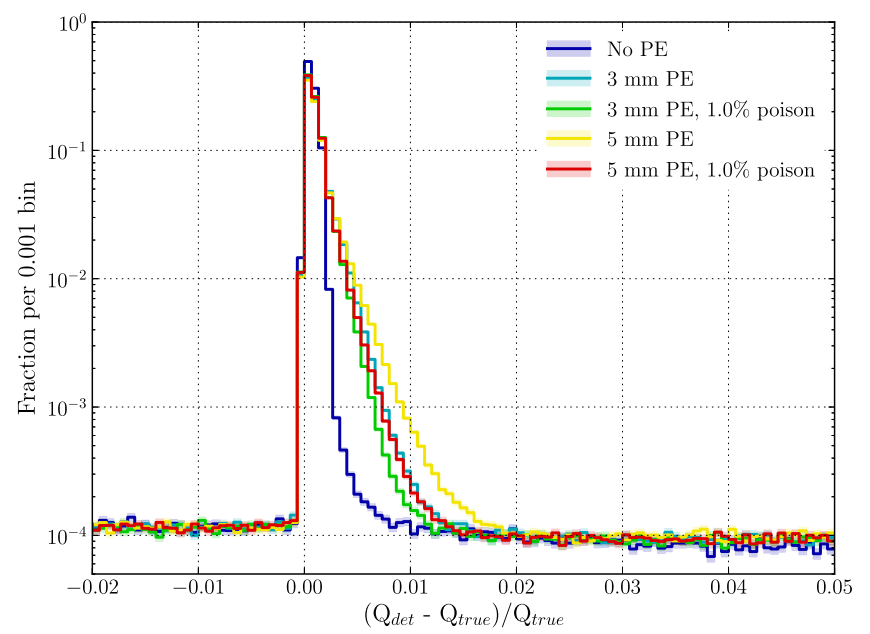

Fig. 23. $Q$ resolution for various combinations of PE thickness and boron poison content. All distributions are normalized to the number of entries.

The improvement after $5 \mathrm{~mm}$ saturates and hence is not shown here. Adding boron poison to the mixture results in the opposite effect. The efficiency gains of $20 \%-40 \%$ can be achieved with the right selection of material thickness and composition.

At the same time, looking at the impact of this methodology on the $Q$ resolution, it becomes apparent that the starting value of the $\delta Q / Q$ width is on the order of $0.5 \%$ and almost doubles with the addition of the backscattering material. However, such a degradation turns out to have minimal impact on the science case, as the $Q$ resolution remains within the instrument requirements [50] of a $\delta Q / Q$ value being below $10 \%$. For this result to make sense, there is an inherent assumption that the detector spatial resolution is appropriate and is not obscuring the effect under discussion.

Looking further into the $Q$ resolution, it is possible to differentiate between spatial and temporal effects that contribute to the broadening of its width, i.e., the $\theta$ resolution $\delta \theta / \theta$ and the time-of-flight resolution $\delta$ tof/tof (see Figs. 24 and 25, respectively). Let it be noted that the intrinsic time resolution of the detector is of a different order of magnitude (approximately below $100 \mathrm{~ns}$ ) and as such, it is entirely negligible for this study. What is of interest here is the time of flight from the sample until the detection event, as this has a much greater impact on the $Q$ resolution of a SANS instrument.

Fig. 24 demonstrates the anticipated broadening of the $\delta \theta / \theta$ width with the addition of $\mathrm{PE}$. The boron poison reduces it at the cost of efficiency gain. The broadening is rather uniform and takes place on both sides of the peak, as expected from the nature of the neutron scattering on the materials of the detector geometry. The small bump appearing on the right side of the distribution is caused by Bragg diffraction.

The shape of the $\delta$ tof/tof distribution (see Fig. 25) is more asymmetric. Negative values correspond to neutrons that scattered and converted early compared to having followed an undisturbed path to the converter. It is this undisturbed path

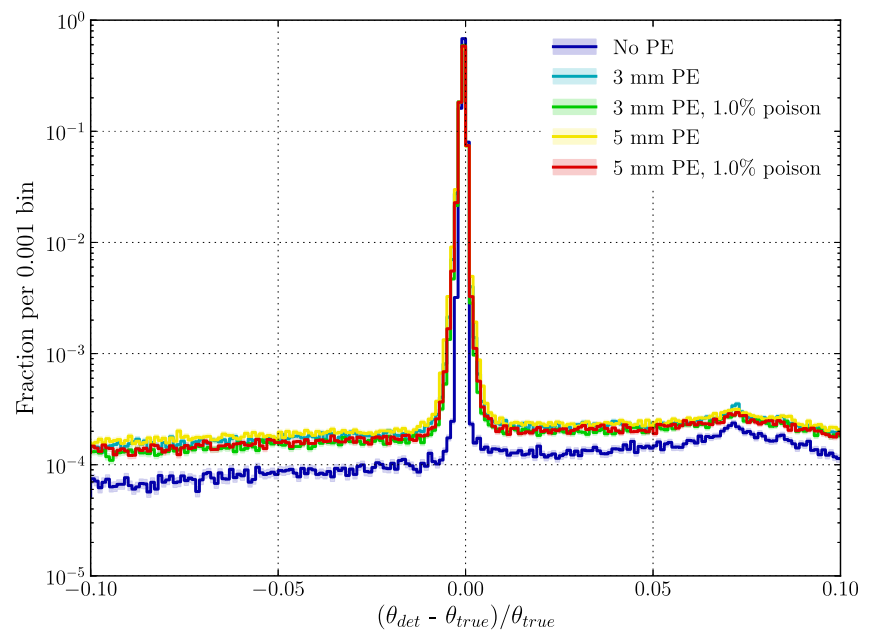

Fig. 24. Polar angle resolution $\delta \theta / \theta$. All distributions have been normalized to their respective number of entries.

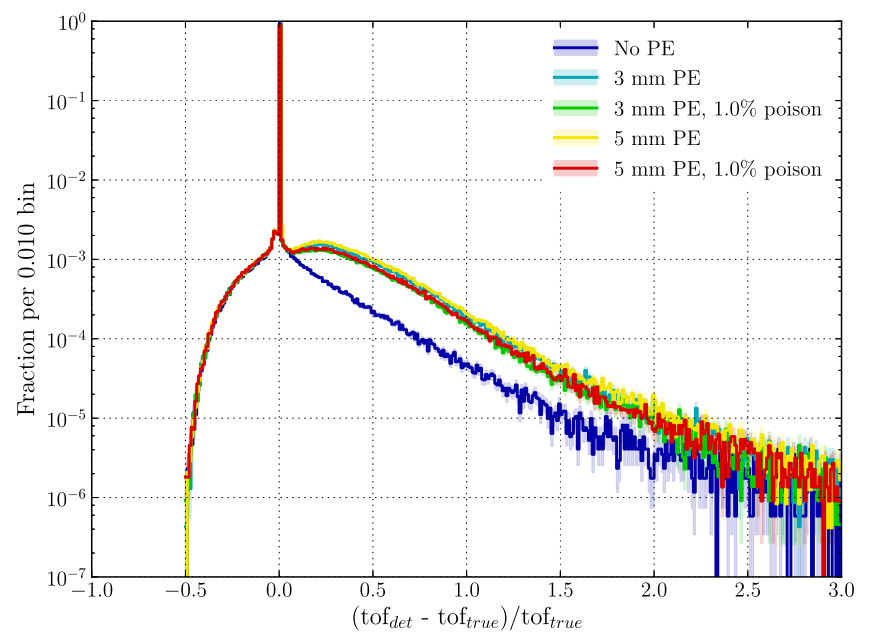

Fig. 25. Time-of-flight $\delta$ tof/tof resolution. All distributions have been normalized to their respective number of entries.

to the boron-carbide layer that is used to determine the tof $f_{\text {true }}$ value based on which the time-of-flight resolution is estimated. The "shoulder" on the left of the distribution reflects the effects of the large geometry, hence it appears both in the presence and absence of PE. The greatest impact from the addition of the $\mathrm{PE}$ is present at higher values, which explains the asymmetric $\delta Q / Q$ distribution. Given the minimal position displacement demonstrated in Fig. 24, larger flight times translate into smaller estimated neutron effective wavelengths, which in turn leads to larger $Q$ values, according to (1). The integral difference between the PE and no PE curves is on the order of a few percent.

In summary, the addition of $\mathrm{PE}$ and absorbing poison in the LoKI tapered detector geometry can benefit its performance significantly, even if applied on a conceptual unoptimized setup. The detection efficiency can be visibly improved, while at the same time, the resolution degradation remains well within the acceptable detector and instrument requirements. 


\section{CONCLUSION}

The concept of enhancing effective detection efficiencies of detectors at neutron instruments by placing a strongly scattering material at their backside was presented and investigated through the analysis of Geant 4 simulations in the scenario of PE placed behind a single-layer thin-film detector. The method shows great promise in the case of neutrons at low angle of incidence (close to perpendicular to the boron coating), with as much as a doubling of efficiency of a single-layer detector in the most ideal scenario. However, care must be taken to keep the potential adverse impact on the spatial and temporal resolutions under control, due to the extra scatterings, either by limiting the amount of scattering material added or possibly via one of the investigated mitigation strategies of poisoning or barrier utilization. The impact on spatial and temporal resolutions in the absence of any such mitigation techniques was respectively seen to be on the order of centimeters and tens of microseconds. The degradation on spatial resolution can be completely contained through the use of barriers where feasible, while the use of poisoning can be used to limit but not completely avoid the impact on the temporal resolution. Thus, the best use case for the method seems to be in detectors for neutron instruments that do not depend on the availability of high timing resolution and that employ costeffective low-efficiency detectors for at least part of their setup. One such indicative use case studied is a futuristic SANS detector geometry, equipped with detectors containing single boron layer converters, for which it is demonstrated that the methodology presented here leads to a tangible performance improvement with a negligible impact on resolution.

\section{REFERENCES}

[1] S. Peggs et al., "ESS conceptual design report," European Spallation Source ERIC, Lund, Sweden, Tech. Rep. ESS 2012-001, 2012. [Online]. Available: http://esss.se/scientific-technical-documentation

[2] S. Peggs et al., "ESS technical design report," European Spallation Source ERIC, Lund, Sweden, Tech. Rep. ESS 2013-001, 2013. [Online]. Available: http://esss.se/scientific-technical-documentation

[3] G. Knoll, Radiation Detection and Measurement, 4th ed. Hoboken, NJ, USA: Wiley, 2012, pp. 519-538.

[4] A. J. Dianoux and G. Lander, Eds., Neutron Data Booklet, 2nd ed. Grenoble, France: Inst. Laue-Langevin, 2003.

[5] O. Kirstein et al., "Neutron position sensitive detectors for the ESS," in Proc. Sci., 2014, p. 029.

[6] R. G. Cooper, "SNS detector plans," Nucl. Instrum. Methods Phys. Res. Section A, Accel., Spectrometers, Detect. Assoc. Equip., vol. 529, pp. 394-398, Aug. 2004.

[7] A. Cho, "Helium-3 shortage could put freeze on low-temperature research," Science, vol. 326, no. 5954, pp. 778-779, 2009.

[8] T. M. Persons and G. Aloise, "Neutron detectors: Alternatives to using helium-3," document GAO-11-753, 2003.

[9] F. Sauli, Gaseous Radiation Detectors: Fundamentals Applications. Cambridge, U.K.: Cambridge Univ. Press, 2014.

[10] F. Sauli, "Principles of operation of multiwire proportional and drift chambers," CERN, Meyrin, Switzerland, Tech. Rep. CERN-77-09, 1977.

[11] G. Charpak, R. Bouclier, T. Bressani, J. Favier, and Č. Zupančič, "The use of multiwire proportional counters to select and localize charged particles," Nucl. Instrum. Methods, vol. 62, no. 3, pp. 262-268, 1968.

[12] R. Charpak and F. Sauli, "High-resolution electronic particle detectors," Annu. Rev. Nucl. Sci, vol. 34, pp. 285-350, Dec. 1984.

[13] K. Zeitelhack, "Search for alternative techniques to Helium-3 based detectors for neutron scattering applications," Neutron News, vol. 23, no. 4 , pp. 10-13, 2012.
[14] International Collaboration for the Development of Neutron Detectors, accessed on Sep. 2016. [Online]. Available: http://www.icnd.org/

[15] C. L. Wang et al., "Wavelength-shifting-fiber scintillation detectors for thermal neutron imaging at SNS," in Proc. IEEE Nucl. Sci. Symp. Conf. Rec., vol. HE4-3. Oct. 2011, pp. 4877-4882.

[16] N. J. Rhodes, E. M. Schooneveld, and R. S. Eccleston, "Current status and future directions of position sensitive neutron detectors at ISIS,' Nucl. Instrum. Methods Phys. Res. Section A, Accel., Spectrometers, Detect. Assoc. Equip., vol. 529, p. 243, Aug. 2004.

[17] A. C. Hannon et al., "Results on disordered materials from the GEneral Materials diffractometer, GEM, at ISIS," Nucl. Instrum. Methods Phys. Res. Section A, Accel., Spectrometers, Detect. Assoc. Equip., vol. 551, no. 1 , p. 88, 2005.

[18] N. J. Rhodes et al., in Proc. ICANS-XV Meeting Int. Collaboration Adv. Neutron Sour., Tsukuba, Japan, 2000, p. 646.

[19] T. Kawasaki et al., "Detector system of the SENJU single-crystal time-of-flight neutron diffractometer at J-PARC/MLF," Nucl. Instrum. Methods Phys. Res. Section A, Accel., Spectrometers, Detect. Assoc. Equip., vol. 735, p. 444, Jan. 2014.

[20] T. Hosoya et al., "Development of a new detector and DAQ systems for iBIX," Nucl. Instrum. Methods Phys. Res. Section A, Accel., Spectrometers, Detect. Assoc. Equip., vol. 600, no. 1, p. 217, 2009.

[21] D. Pfeiffer et al., "First measurements with new high-resolution gadolinium-GEM neutron detectors," J. Instrum., vol. 11, p. P05011, May 2016.

[22] P. P. Deen et al., "A design study of VOR: A versatile optimal resolution chopper spectrometer for the ESS," in Proc. EPJ Web Conf., vol. 83. 2015, p. 03002.

[23] C. Höglund et al., " $\mathrm{B}_{4} \mathrm{C}$ thin films for neutron detection," J. Appl. Phys, vol. 111, no. 10, p. 104908, 2012.

[24] K. Andersen et al., " ${ }^{10} \mathrm{~B}$ multi-grid proportional gas counters for large area thermal neutron detectors," Nucl. Instrum. Methods Phys. Res. Section A, Accel., Spectrometers, Detect. Assoc. Equip., vol. 720, pp. 116-121, 2013.

[25] A. Khaplanov et al., "Investigation of gamma-ray sensitivity of neutron detectors based on thin converter films," J. Instrum., vol. 8, p. P10025, Oct. 2013.

[26] F. Piscitelli and P. van Esch, "Analytical modeling of thin film neutron converters and its application to thermal neutron gas detectors," $J$. Instrum., vol. 8, p. P04020, Apr. 2013.

[27] E. Piesch, "Progress in albedo neutron dosimetry," Nucl. Instrum. Methods Phys. Res. Section A, Accel., Spectrometers, Detect. Assoc. Equip., vol. 145, no. 3, pp. 613-619, 1977.

[28] E. Piesch, "Albedo neutron dosimetry," Int. J. Appl. Radiation Isotopes, vol. 33, no. 11, pp. 1061-1076, 1982.

[29] E. Piesch and B. Burgkhardt, "Albedo neutron dosimetry," Radiation Protection Dosimetry, vol. 10, nos. 1-4, pp. 175-188, 1985.

[30] S. Glasstone, Principles of Nuclear Reactor Engineering (Principles Of Nuclear Reactor Engineering). New York, NY, USA: van Nostrand, 1955.

[31] Z. W. Bell, W. G. West, K. R. Pohl, and L. van den Berg, "Monte Carlo analysis of a mercuric iodide neutron/gamma detector," IEEE Trans. Nucl. Sci., vol. 52, no. 5, pp. 2030-2034, Oct. 2005.

[32] Z. W. Bell and L. A. Boatner, "Neutron absorption detector," U.S. Patent App. 12228661, Feb. 18, 2010. [Online]. Available: https://www.google.com/patents/US20100038551

[33] S. Agostinelli et al., "Geant4-A simulation toolkit," Nucl. Instrum. Methods Phys. Res. Section A, Accel., Spectrometers, Detect. Assoc. Equip., vol. 506, no. 3, pp. 250-303, 2003.

[34] J. Allison et al., "Geant4 developments and applications," IEEE Trans. Nucl. Sci., vol. 53, no. 1, pp. 270-278, Feb. 2006.

[35] J. Allison et al., "Recent developments in Geant4," Nucl. Instrum. Methods Phys. Res. Section A, Accel., Spectrometers, Detect. Assoc. Equip., vol. 835, pp. 186-225, Nov. 2016.

[36] T. Kittelmann, I. Stefanescu, K. Kanaki, M. Boin, R. Hall-Wilton, and K. Zeitelhack, "Geant4 based simulations for novel neutron detector development," J. Phys., Conf. Ser., vol. 513, no. 2, p. 022017, 2014.

[37] F. Piscitelli et al., "Study of a high spatial resolution ${ }^{10} \mathrm{~B}$-based thermal neutron detector for application in neutron reflectometry: The multi-blade prototype," J. Instrum., vol. 9, no. 3, p. P03007, 2014.

[38] J. Birch et al., "In-beam test of the boron-10 multi-grid neutron detector at the IN6 time-of-flight spectrometer at the ILL,"J. Phys., Conf. Ser., vol. 528, no. 1, p. 012040, 2014. 
[39] I. Stefanescu et al., "A ${ }^{10}$ B-based neutron detector with stacked MultiWire Proportional Counters and macrostructured cathodes," J. Instrum., vol. 8, no. 12, p. P12003, Dec. 2013.

[40] J. L. Lacy, A. Athanasiades, C. S. Martin, L. Sun, G. J. Vazquez-Flores, and T. D. Lyons, "Boron-coated straw detectors: A novel approach for helium-3 neutron detector replacement," in Proc. IEEE Nucl. Sci. Symp. Med. Imag. Conf., Oct. 2010, pp. 3971-3975.

[41] M. Henske et al., "The ${ }^{10} \mathrm{~B}$ based Jalousie neutron detector-An alternative for ${ }^{3} \mathrm{He}$ filled position sensitive counter tubes," Nucl. Instrum. Methods Phys. Res. Section A, Accel., Spectrometers, Detect. Assoc. Equip., vol. 686, pp. 151-155, Sep. 2012.

[42] I. Stefanescu et al., "Development of a novel macrostructured cathode for large-area neutron detectors based on the ${ }^{10} \mathrm{~B}$-containing solid converter," Nucl. Instrum. Methods Phys. Res. Section A, Accel., Spectrometers, Detect. Assoc. Equip., vol. 727, pp. 109-125, Nov. 2013.

[43] B. M. van der Ende, J. Atanackovic, A. Erlandson, and G. Bentoumi, "Use of GEANT4 vs. MCNPX for the characterization of a boron-lined neutron detector," Nucl. Instrum. Methods Phys. Res. Section A, Accel., Spectrometers, Detect. Assoc. Equip., vol. 820, pp. 40-47, Jun. 2016.

[44] T. Kittelmann and M. Boin, "Polycrystalline neutron scattering for Geant4: NXSG4," Comput. Phys. Commun., vol. 189, pp. 114-118, Apr. 2015.

[45] A. J. Konig et al., "Status of the JEFF nuclear data library," J. Korean Phys. Soc., vol. 59, no. 2, pp. 1057-1062, 2011.
[46] D. B. Pelowitz et al., "MCNPX 2.7.0 extensions," Los Alamos Nat. Lab., New Mexico, NM, USA, Tech. Rep. LA-UR-11-02295, 2011.

[47] R. E. MacFarlane et al., "The NJOY nuclear data processing system, version 2012," Los Alamos Nat. Lab., New Mexico, NM, USA, Tech. Rep. LA-UR-12-27079, 2012.

[48] B. Wiegel and A. V. Alevra, "NEMUS-The PTB neutron multisphere spectrometer: Bonner spheres and more," Nucl. Instrum. Methods Phys. Res. Section A, Accel., Spectrometers, Detect. Assoc. Equip., vol. 476, nos. $1-2$, pp. 36-41, 2002.

[49] J. Keinert, M. Mattes, and E. Sartori, "JEF-1 scattering law data," IKE Stuttgart, Stuttgart, Germany, Tech. Rep. JEF Report 2/JEF/DOC 41.2, 1984.

[50] A. J. Jackson et al., "LoKI-A broad band high flux SANS instrument for the ESS," in Proc. 21th ICANS, 2015, pp. 1-9.

[51] L. A. Feigin and D. Svergun, Structure Analysis by Small-Angle $X$-Ray and Neutron Scattering. New York, NY, USA: Springer, 1987.

[52] K. Kanaki et al., "A novel small-angle neutron scattering detector geometry," J. Appl. Crystallogr., vol. 46, pp. 1031-1037, Aug. 2013.

[53] K. Kanaki et al., "A novel small-angle neutron scattering detector geometry (corrigendum)," J. Appl. Crystallogr., vol. 46, p. 1528 , Sep. 2013. 